\title{
Formation of Lithium Titanate Hydrate Nanosheets: Insight into a Two-Dimension Growth Mechanism by in Situ Raman
}

Hsien-Chieh Chiu, ${ }^{\dagger}$ Xia Lu, ${ }^{\dagger}$ Samir Elouatik, ${ }^{\dagger}$ Karim Zaghib, ${ }^{\S}$ and George P. Demopoulos $*{ }^{\dagger}$

${ }^{\dagger}$ Materials Engineering, McGill University, Montréal, Québec H3A 0C5, Canada

¥Département de Chimie, Université de Montréal, C.P. 6128, Succursale Centre-ville, Montréal, Québec, H3C 3J7, Canada

§ Institut de recherche d'Hydro-Québec (IREQ), Varennes, Québec J3X 1S1, Canada

* Email: george.demopoulos@ @mcgill.ca. Tel: +15143982046. Fax: +15143984492. 


\section{Derivation of Johnson-Mehl-Avrami-Kolmogorov (JMAK) model for anisotropic growth}

The modified JAMK kinetic model for anisotropic growth was derived directly from

Kooi's work. ${ }^{1-2}$ Only a special case of $2 \mathrm{D}$ growth in 3D space with continuous nucleation at constant rate was considered in our study.

The kinetics of isothermal phase transformation proceeding via nucleation and subsequent growth are generally described by the JMAK theory, which states that the fraction of transformed space $x$ is related to the extended fraction $x_{\text {extended }}$ according to:

$$
x(t)=1-\exp \left[-x_{\text {extended }}(t)\right]
$$

where the extended fraction $x_{\text {extended }}(t)$ obeys

$$
x_{\text {extended }}(t)=\int_{0}^{t} I(\tau) V(t-\tau) d \tau
$$

With $I$ the nucleation rate per unit of untransformed space; and $\mathrm{V}$ the extended space of a single transformed grain at time $t ; \tau$ is the nucleation time of that transformed grain.

According to the first assumption of constant nucleation rate, the total number of nuclei formed per unit of untransformed space with dimension, $\mathrm{d}_{\mathrm{s}}$, at a given temperature, behaves as a linear equation i.e. $N=N_{0} t$, which define the initial nucleation rate $\mathrm{N}_{0}$ with temperature dependency becomes:

$$
N(t, T)=N_{0} t \exp \left(\frac{-E_{n}}{k T}\right)
$$

From which the nucleation rate can be easily calculated as: 


$$
I(t, T)=\frac{d N(t, T)}{d t}=N_{0} \exp \left(\frac{-E_{n}}{k T}\right)
$$

Then the second assumption of linear growth of grain for the interfacial reaction process is reasonable for the present system as it involves crystallization from an amorphous precursor with highly disordered interface. ${ }^{3-4}$ The growth rate in this case can be formulated as the difference in forward and backward reaction rates:

$$
G(T)=G_{0} \exp \left(\frac{-E_{g}}{k T}\right)\left[1-\exp \left(\frac{\Delta G}{k T}\right)\right]
$$

where $E_{g}$ is the activation energy for growth $\left(E_{g}>0\right)$ and $\Delta G$ is the difference in Gibbs free energy per atom of the phase, after and before the phase transformation. The transformation would be spontaneous if $\Delta \mathrm{G}<0$. If the driving force $-\Delta \mathrm{G}$ is larger compared to thermal excitation (kT 0.03 eV), the backward reaction rate can be neglected:

$$
G(T)=G_{0} \exp \left(\frac{-E_{g}}{k T}\right)\left[1-\exp \left(\frac{\Delta G}{k T}\right)\right] \rightarrow G(T)=G_{0} \exp \left(\frac{-E_{g}}{k T}\right)
$$

Now let us consider that 2D plates with thickness $w$ have equal probability to grow in three orthogonal directions in 3D space. The average length the grains are able to grow before their growth is blocked is the characteristic length $\overline{L_{l}}$ which is expressed as:

$$
\overline{L_{\imath}}=\frac{C}{\sqrt[3]{N\left(\tau^{a}\right)}}
$$

By replacing the $V(t-\tau)$ in Eq. (S1b) by the width $w$, times the square of characteristic length $\overline{L_{l}}$ as

$$
V(t-\tau)=w{\overline{\left(L_{l}\right)^{2}}}^{2}=\frac{w C^{2}}{\left(N \tau^{a}\right)^{2 / 3}}
$$

and then inserting it as well as I(t,T) from Eq. (S3) back into Eq. (S1b) we obtain: 


$$
x_{\text {extended }}(t)=\int_{t^{*}}^{t} a N \tau^{a-1} \frac{w C^{2}}{\left(N \tau^{a}\right)^{2 / 3}} d \tau
$$

in which the $\mathrm{t}^{*}$ is defined as the time when growth stops because of blocking from a neigbouring 2D plate:

$$
G t^{*}=\frac{C}{\sqrt[3]{N\left(t^{*}\right)}} \rightarrow t^{*}=\sqrt[1+3]{\frac{C^{3}}{N G^{3}}}
$$

By inserting $\mathrm{t}^{*}$ into the integration result of Eq. (S6), the modified transformed fraction for two-dimensional anisotropic growth in 3D space is obtained as:

$$
x_{\text {extended }}(t)=3 w C^{2}\left[N\left(t-t^{*}\right)\right]^{1 / 3}
$$

where the Avrami exponent is equal to $1 / 3$.

In the limitation case of $\mathrm{t}>\mathrm{t}^{*}$, Eq. (S1a) becomes

$$
x(t)=1-\exp \left[-x_{\text {extended }}(t)\right]=1-\exp \left[3 w C^{2}\left[N\left(t-t^{*}\right)^{a}\right]^{1 / 3}\right]
$$

Employing Avrami plot analysis in the form of $\ln \{-\ln [1-\mathrm{x}(\mathrm{t})]\}$ vs. $\ln (\mathrm{t})$ we get:

$$
\begin{gathered}
\ln (-\ln [1-x(t)])=\ln \left(x_{\text {extended }}(t)\right)=\ln \left[3 w C^{2}\left[N\left(t-t^{*}\right)\right]^{\frac{1}{3}}\right] \\
\ln (-\ln [1-x(t)])=\ln \left(3 w C^{2}(N)^{\frac{1}{3}}\right)+\frac{1}{3} \ln \left(t-t^{*}\right)
\end{gathered}
$$

now replacing $\mathrm{N}$ in Eq. (S7) by Eq. (S2), the intercept in Avrami plot becomes

$$
\begin{gathered}
\ln \left(3 w C^{2}(N)^{\frac{1}{3}}\right)=\ln \left(3 w C^{2}\right)+\frac{1}{3} \ln \left[N_{0} \exp \left(\frac{-E_{n}}{k T}\right)\right] \\
\rightarrow \ln \left(3 w C^{2}\right)+\frac{1}{3} \ln \left(N_{0}\right)+\frac{1}{3}\left(\frac{-E_{n}}{k T}\right)
\end{gathered}
$$


In the Arrhenius plot analysis, its slope turns out to be $\frac{1}{3}\left(\frac{-E_{n}}{k T}\right)$

\section{Estimation of residual strain from Raman measurements}

The quantitative estimation of residual strain is conducted via calculating the peak position difference between reference LTH material and the different LTH crystallization products. Precise Peak position was determined by applying pseudo-Voigt type ${ }^{5}$ fitting on one of the significant $\mathrm{Ag}$ bands at $433 \mathrm{~cm}^{-1}$. The referenced LTH material was prepared by $240{ }^{\circ} \mathrm{C}$ hydrothermal treatment ${ }^{6-8}$ and is considered as the "perfect" crystal without residual strain, i.e. only relative residual train is obtained by this method. In the end, the residual strain can be calculated according to equation ${ }^{9-10}$ :

$$
\frac{\Delta \omega}{\omega_{0}}=-K \frac{a-a_{0}}{a_{0}}=-K \frac{\Delta a}{a_{0}}
$$

where $\omega_{0}$ is the Raman shift $433 \mathrm{~cm}^{-1}$ of referenced LTH; $\Delta \omega$ is the difference between the reference $433 \mathrm{~cm}^{-1}$ and the crystallized LTH sample at specific time; $\mathrm{a}_{0}$ is the lattice constant of referenced LTH; only $b$-axis with $16.7023(2) \AA$ is employed for simplicity; a is the lattice constant of $b$-axis of the corresponding LTH samples; and $K$ is a constant containing the Grüneisen parameter ${ }^{2}$. 


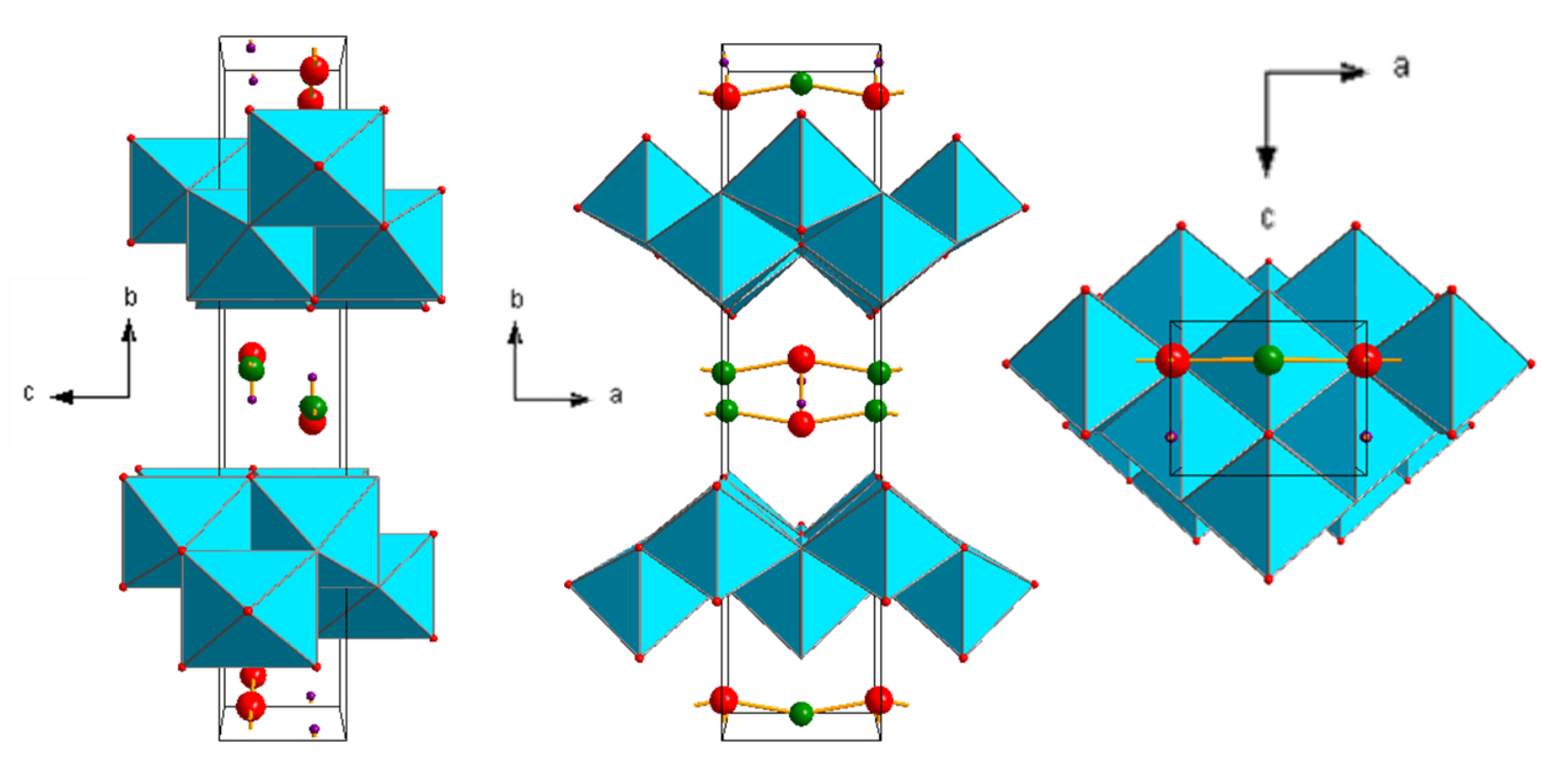

Figure S1. The schematic of the proposed orthorhombic lepidocrocite LTH (space group $\mathrm{Cmcm}$ ) model with lattice parameters: $a$-axis $=3.8020 \AA$; $b$ axis $=16.6729 \AA$; and $c$-axis $=3.0093 \AA$. Red balls are oxygen atoms, green represents lithium atoms and the small purple balls are hydrogen. 

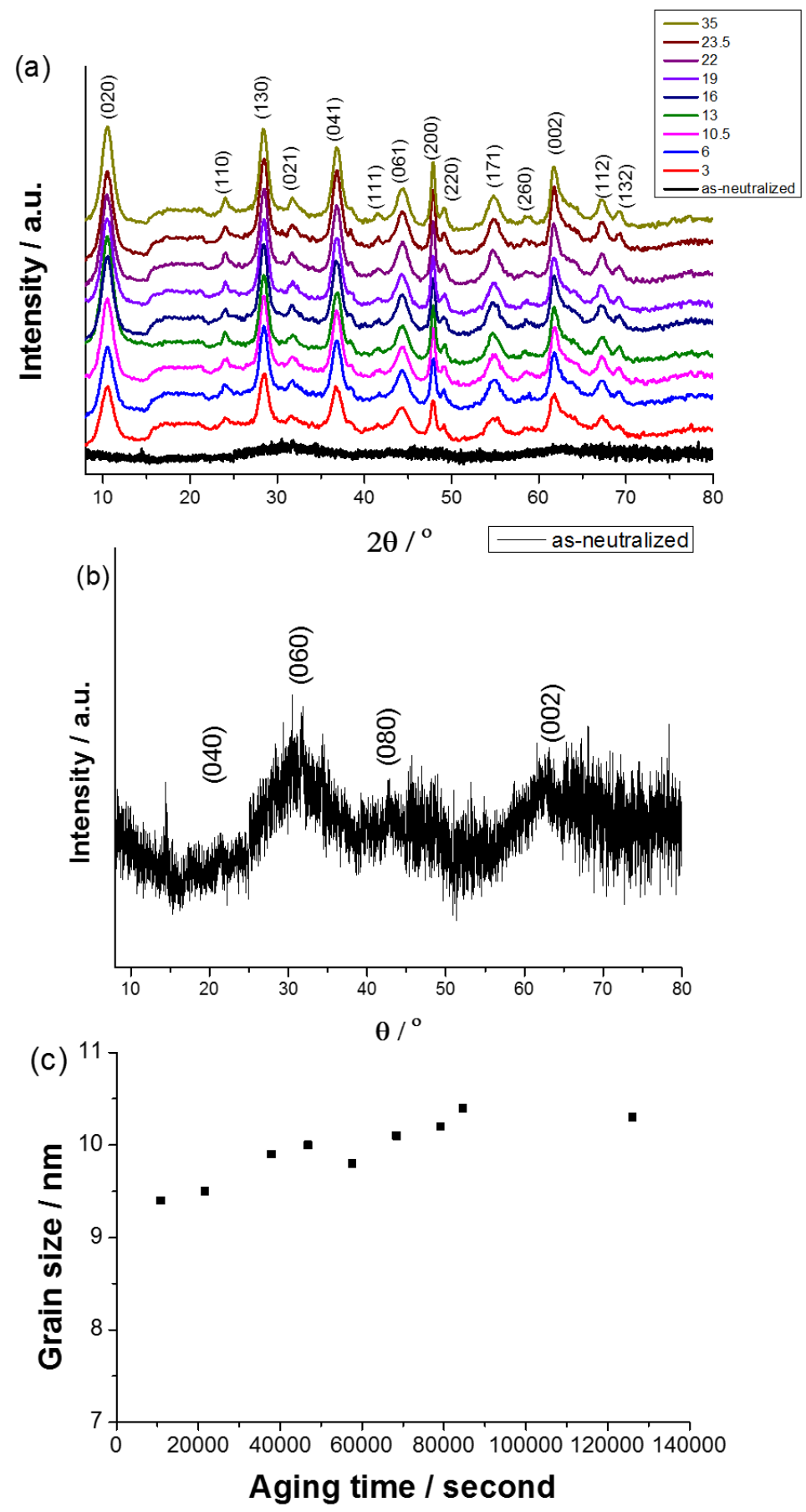

Figure S2. (a) Time evolution of PXRD patterns of isothermally aged samples, (b) the magnified pattern of as-neutralized precipitate, and (c) the grain size calculated by Scherrer equation according to the PXRD patterns in (a). 
(a)

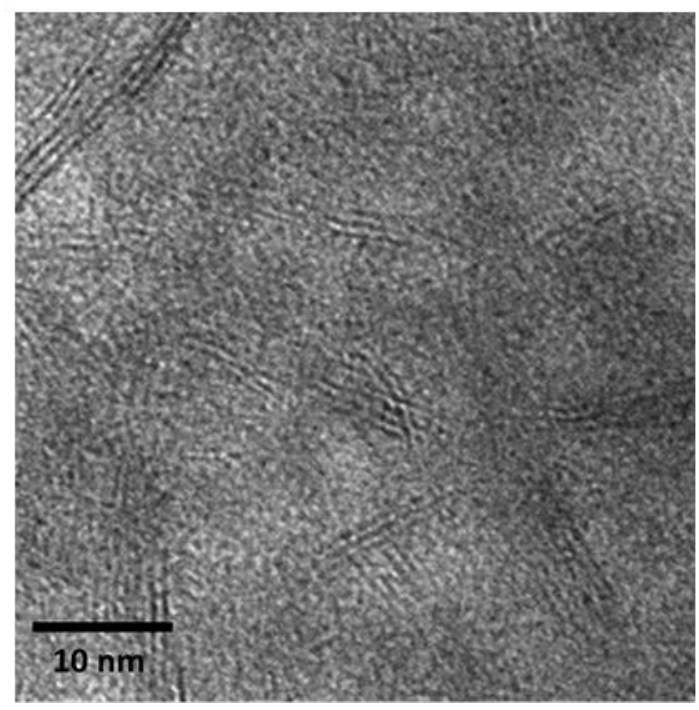

(b)

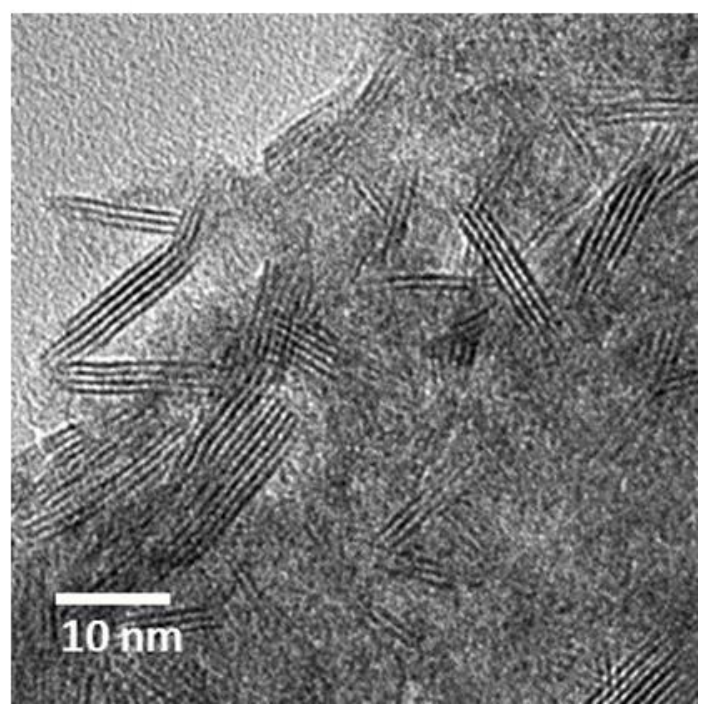

Figure S3. Nanoscale curved features of (a) as-neutralized precursor precipitate and (b) isothermally crystallized LTH at $90{ }^{\circ} \mathrm{C}$ for 3 hours. 

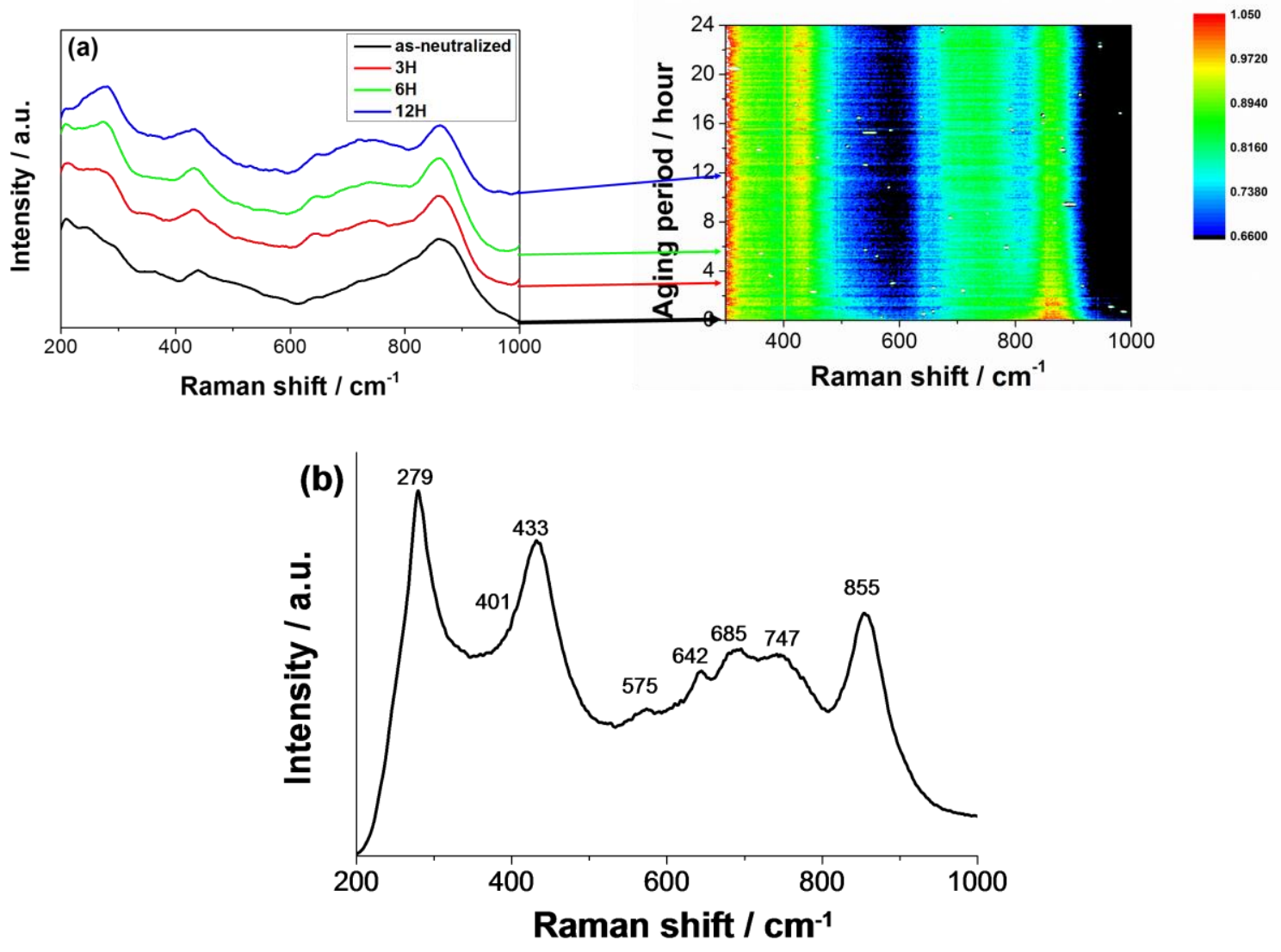

Figure S4. (a) Schematic diagram describing the obtainment of the Raman spectra on the left by cross sectioning of the in situ Raman contour plots on the right; (b)the Raman spectrum of the reference LTH phase prepared by hydrothermal process at $240{ }^{\circ} \mathrm{C}$ for 48 hours. 


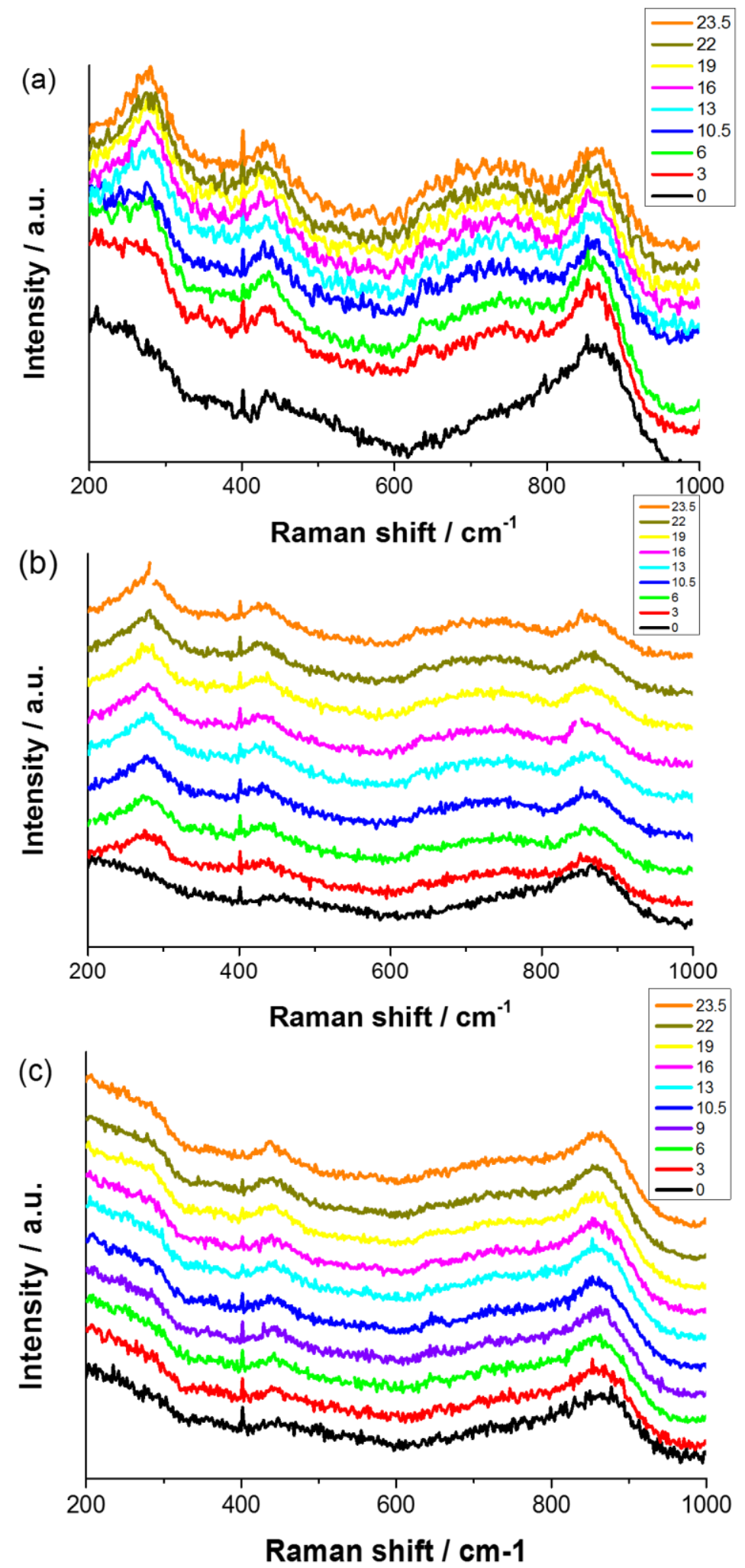

Figure S5. In-situ Raman measurements during isothermal crystallization at (a) 90 (b) 75 and (c) $50{ }^{\circ} \mathrm{C}$. 


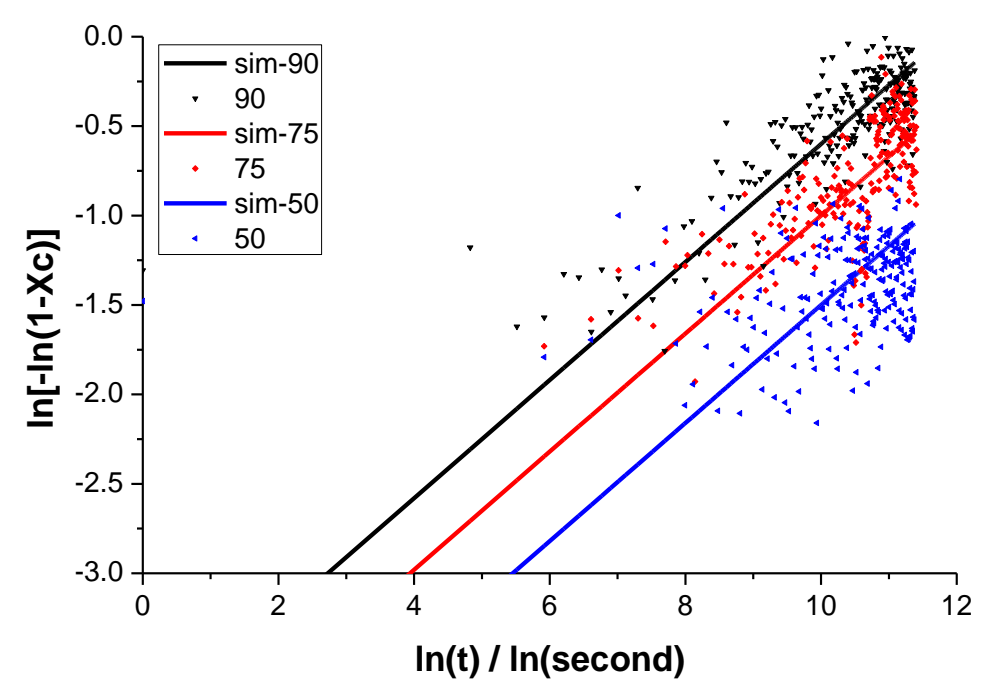

Figure S6. The Avrami plot $(\ln [-\ln (1-x)] v s \ln (t))$ of LTH growth in isothermal condition at (a) 90 ${ }^{\circ} \mathrm{C}$ (black), (b) $75{ }^{\circ} \mathrm{C}$ (blue) and (c) $50{ }^{\circ} \mathrm{C}$ (red). Please note the Avrami exponent $=1 / 3$.

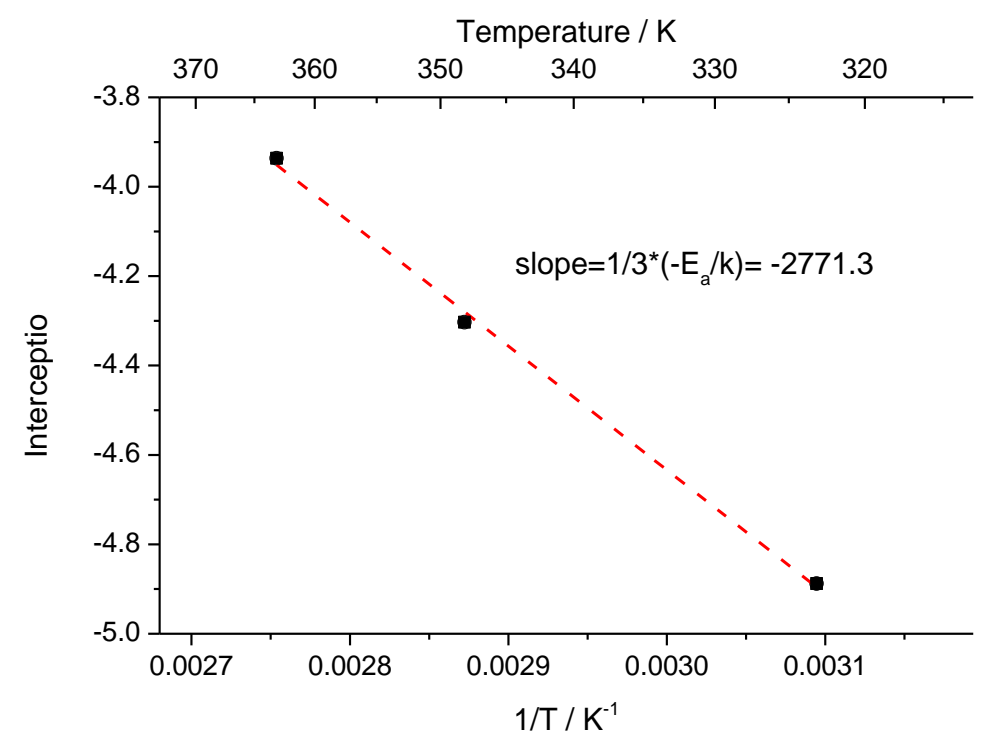

Figure S7. The Arrhenius plot calculated from the intercept on Y-axis of the Avrami plot (Figure S6), in which the slope corresponds to the activation energy $\mathrm{E}_{\mathrm{n}}$ in the form of $\frac{1}{3}\left(\frac{-E n}{k}\right)$ where $\mathrm{k}$ is Boltzmann's constant. 

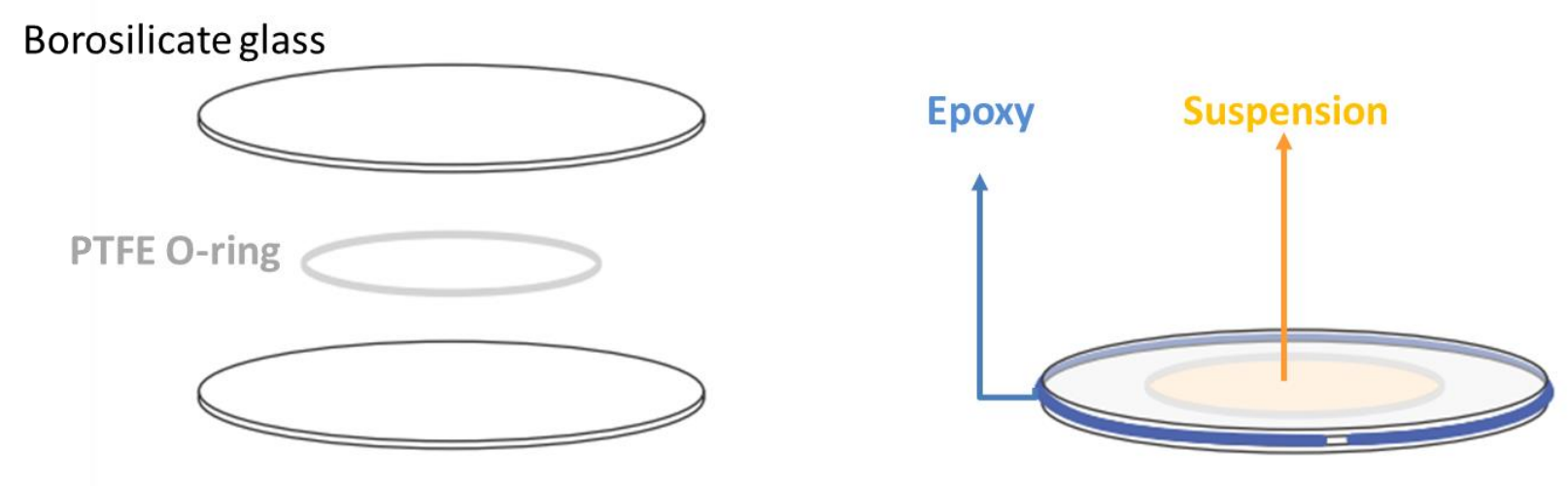

Figure S8. The schematic diagram of the in situ reactor for isothermal crystallization of LTH

\section{References}

1. $\quad$ Kooi, B. J., Phys. Rev. B: Condens. Matter Mater. Phys. 2006, 73 (5), 054103.

2. $\quad$ Kooi, B. J., Phys. Rev. B: Condens. Matter Mater. Phys. 2004, 70 (22), 224108.

3. $\quad$ Kingary, W.; Bowen, H.; Uhlmann, D., Introduction to Ceramics. 1976.

4. $\quad$ Knrpnrnrcr, R. J., Am. Mineral. 1975, 60, 798-814.

5. Thompson, P.; Cox, D. E.; Hastings, J. B., J. Appl. Cryst. 1987, 20 (2), 79-83.

6. $\quad$ Shirpour, M.; Cabana, J.; Doeff, M., Chem. Mater. 2014, 26 (8), 2502-2512.

7. $\quad$ Sugita, M.; Tsuji, M.; Abe, M., Bull. Chem. Soc. Jpn. 1990, 63 (7), 1978-1984.

8. $\quad$ Li, N.; Mei, T.; Zhu, Y.; Wang, L.; Liang, J.; Zhang, X.; Qian, Y.; Tang, K., CrystEngComm 2012, 14 (20), 6435-6440.

9. $\quad$ Arguirov, T.; Mchedlidze, T.; Kittler, M.; Rolver, R.; Berghoff, B.; Forst, M.; Spangenberg, B., Appl. Phys. Lett. 2006, 89 (5), 053111.

10. Wang, R. P.; Zhou, G. W.; Liu, Y. L.; Pan, S. H.; Zhang, H. Z.; Yu, D. P.; Zhang, Z., Phys. Rev. B: Condens. Matter Mater. Phys. 2000, 61 (24), 16827-16832. 